Supporting Information

\title{
A Covalent Organic Polymer as a Carborane Carrier for Imaging-Facilitated Boron Neutron Capture Therapy
}

Yaxin Shi ${ }^{1 *}$, Qiang $\mathrm{Fu}^{2 *}$, Jiyuan $\mathrm{Li}^{1}, \mathrm{Hui} \mathrm{Liu}^{1}$, Zizhu Zhang ${ }^{4}$, Tong Liu ${ }^{4}$, Zhibo Liu ${ }^{1,3 *}$

${ }^{1}$ Radiochemistry and Radiation Chemistry Key Laboratory of Fundamental Science, Beijing National Laboratory for Molecular

Sciences, College of Chemistry and Molecular Engineering, Peking University, Beijing, 100871, China

${ }^{2}$ The Centre of Nanoscale Science and Technology and Key Laboratory of Functional Polymer Materials, Institute of Polymer Chemistry, College of Chemistry, Nankai University, Tianjin 300071, China.

${ }^{3}$ Peking University-Tsinghua University Center for Life Sciences, Beijing, 100871, China

${ }^{4}$ Beijing Capture Tech Co., Ltd., Beijing, 102413 , China.

*E-mail: zbliu@pku.edu.cn 


\section{MATERIALS AND METHODS}

Chemicals and Materials. 5,10,15,20-Tetrakis(4-aminophenyl) porphyrin (TAPP) and anhydrous dimethyl sulfoxide (DMSO) were obtained from J\&K Chemical Co., Ltd., and the cell counting kit-8 (CCK-8) was purchased from Biyuntian Biotechnology Institute. ${ }^{64} \mathrm{Cu}$ was obtained from Beijing Cancer Hospital, where it was produced on a cyclotron via a ${ }^{64} \mathrm{Ni}(\mathrm{p}, \mathrm{n}){ }^{64} \mathrm{Cu}$ reaction and purified to yield ${ }^{64} \mathrm{Cu}$ chloride. 1,2Distearoyl-sn-glycero-3-phosphoethanolamine-N-[amino(polyethylene-glycol)-2000] (DSPE-PEG, molecular weight 2000) was obtained from Biomatrik Inc. All organic solvents, such as methanol $(\mathrm{MeOH})$, dichloromethane (DCM), ethanol (EtOH), acetonitrile $(\mathrm{MeCN})$, chloroform $\left(\mathrm{CHCl}_{3}\right)$, tetrahydrofuran (THF), 1,2-dichlorobenzene (o-DCB) and butyl alcohol (n-BuOH), were analytical grade reagents used without further purification. All of the glassware was dried at $120^{\circ} \mathrm{C}$ overnight and cooled under vacuum before use.

Characterization. Transmission electron microscopy (TEM) was performed on a JEM-2100F microscope (JEOL Ltd., Japan). Nuclear magnetic resonance (NMR) spectra were recorded on Bruker AVANCE spectrometers (400 $\mathrm{MHz})$. The hydrodynamic sizes and zeta potentials were determined with a Nanobrook Omni (New York, U.S.A.). Powder X-ray diffraction (XRD) measurements were carried out by using a Rigaku Ultima IV X-ray powder diffractometer with $\mathrm{Cu} \mathrm{K} \alpha$ radiation $(\lambda=$ $1.54056 \AA$ ) over the range of $2 \theta=2.1-30.0^{\circ}$ with a step size of $0.02^{\circ}$ and 0.4 s per step. UV-Vis spectrum measurements were performed using a UV3600 Plus. Fluorescence images were obtained with a Nikon A1R confocal laser scanning microscope. Fourier transform infrared (FT-IR) resonance spectra were obtained on a Spectrum Spotlight 200 FT-IR spectrometer. Inductively coupled plasma-atomic emission spectroscopy (ICP-AES) was carried out on Prodigy 7 (Leeman). PET images were taken with a micro PET (Siemens Medical Solutions USA, Inc.). 
Drug Loading and Release Behavior of COP-5T. TAPP was dissolved in dimethylformamide (DMF) at a series of different concentrations. The maximum absorption wavelength of TAPP was $420 \mathrm{~nm}$, and at this wavelength the DF5T block had no UV absorption. The absorbance of the TAPP solution at different concentrations was measured. The concentration of TAPP was determined by standard concentration curves. Each analysis was conducted in triplicate. Carborane loading (12.31\% and $15.93 \%)$ and encapsulation efficiency (5.49\% and 9.04\%) of DSPE-B and DSPEBCOP-5T were calculated respectively from the following equations by ICP-AES measurements.

Carborane loading $(\%)=($ amount of loaded carborane in $\mathrm{mg}) /($ amount of coating reagent in $\mathrm{mg}) \times 100 \%$

Encapsulation efficiency $(\%)=($ amount of loaded carborane in $\mathrm{mg}) /($ amount of carborane added in $\mathrm{mg}) \times 100 \%$

Typically, phosphate buffer $(20 \mathrm{mM}, \mathrm{pH}=8.0-8.5)$ containing $100 \mu \mathrm{g} / \mathrm{mL}$ DSPECOP-5T solution was continuously stirred at $4^{\circ} \mathrm{C}$. At different incubation times, the residual carborane was separated by ultrafiltration and washed with phosphate-buffered saline (PBS). The samples were further processed for ICP-AES analysis to determine the release properties of COP-5T.

Cell Viability Assay. The in vitro cell viability of drug-loaded COP-5T was assessed with the CCK8 proliferation assay following the manufacturer's protocol. ${ }^{1-2}$ Briefly, adherent 4T1 cells were incubated with different concentrations of DSPE-COP-5T and DSPE-BCOP-5T. After $24 \mathrm{~h}$, the cells were washed with fresh medium and further incubated for $24 \mathrm{~h}$ to run the CCK8 assay test to determine the relative cell viability.

Cellular Internalization of FITC-DSPE-BCOP-5T. 4T1 cells were incubated with FITC-DSPE-BCOP-5T for different lengths of time $(2 \mathrm{~h}$ and $24 \mathrm{~h})$ and then washed with PBS three times followed by labeling with 4,6-diamino-2-phenylindole (DAPI). All samples were imaged with a Nikon A1R confocal laser scanning microscope. 
Histology. On day 20, the mice from different groups were randomly selected and dissected. The heart, liver, spleen, lung and kidney were excised and fixed in $4 \%$ paraformaldehyde, embedded in paraffin, and cut into 5- $\mu \mathrm{m}$ sections for analysis. The H\&E staining images were acquired by optical microscopy.

Statistical Analysis. Statistical analyses were performed using Prism, version 6.0 (GraphPad Software, Inc.) and Excel (Microsoft). Data were analyzed using the unpaired, 2-tailed Student's t-test. Differences at the 95\% confidence level $(\mathrm{P}<0.05)$ were considered to be statistically significant. 


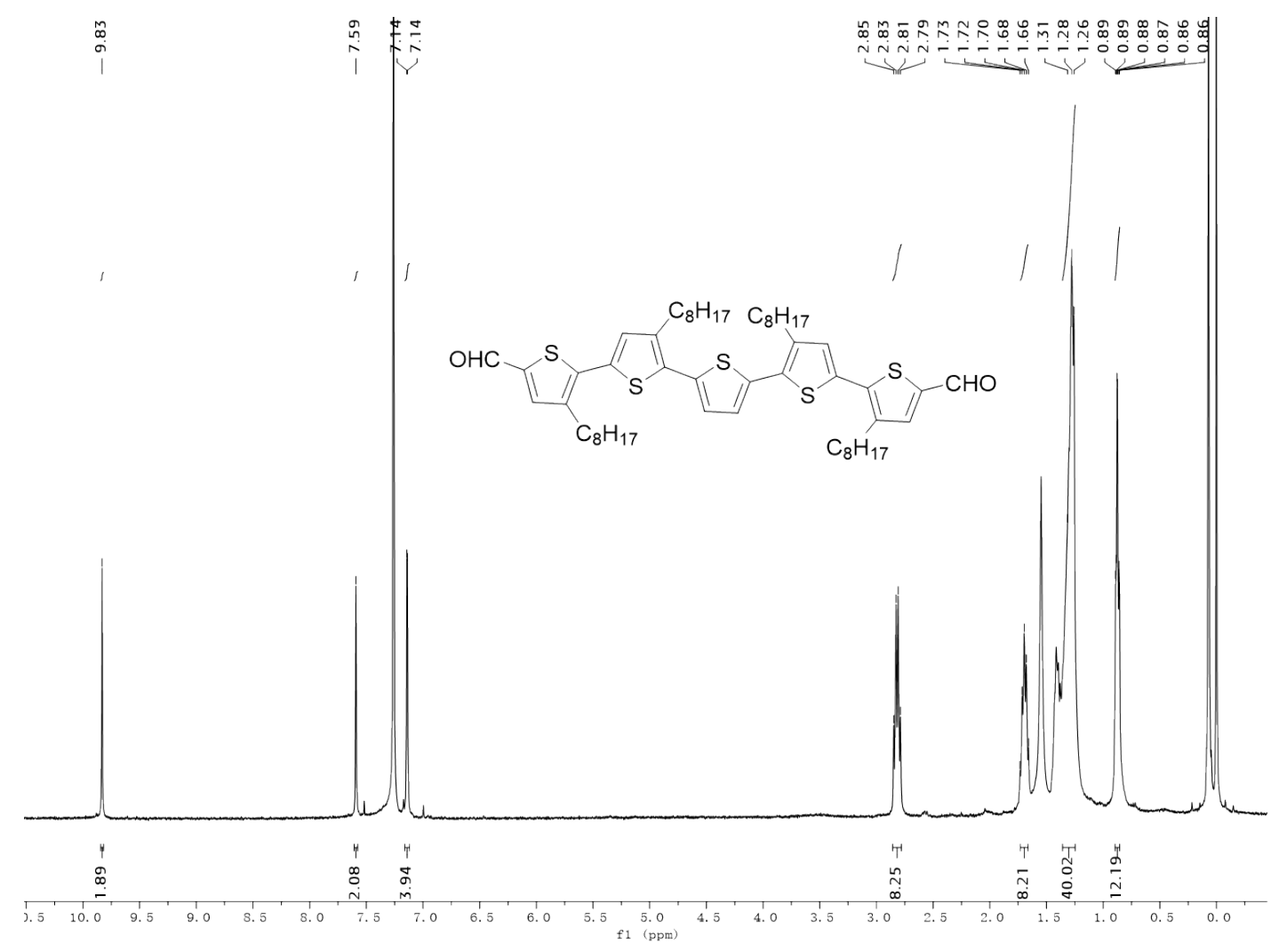

Figure S1. ${ }^{1} \mathrm{H}$ NMR spectrum of DF5T in $\mathrm{CDCl}_{3}$. 
a)

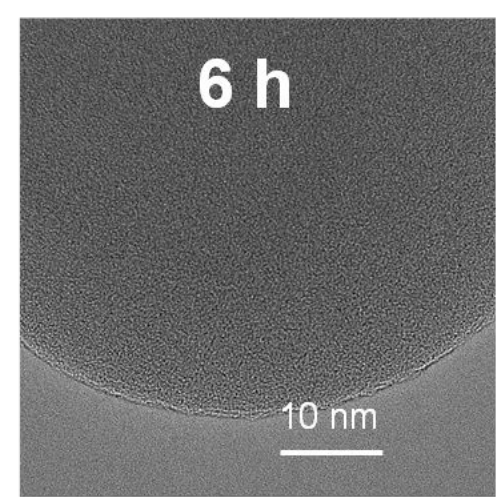

b)

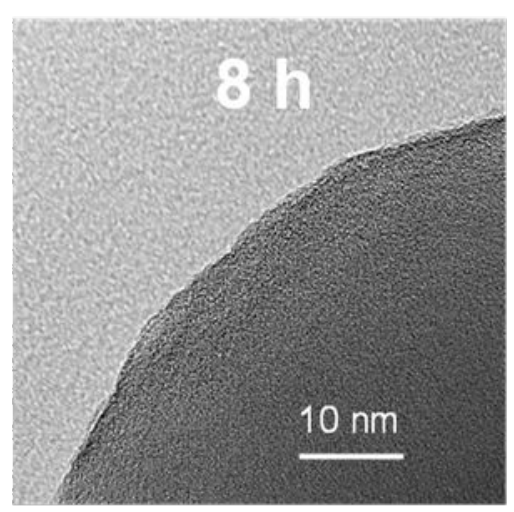

c)

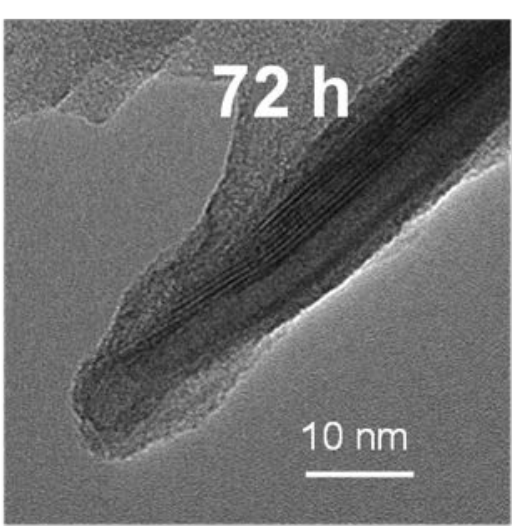

Figure S2. TEM images of COP-5T with different polymerization times in large scale. (a) 6 hours. (b) 8 hourS. (c) 72 hours. 
a)

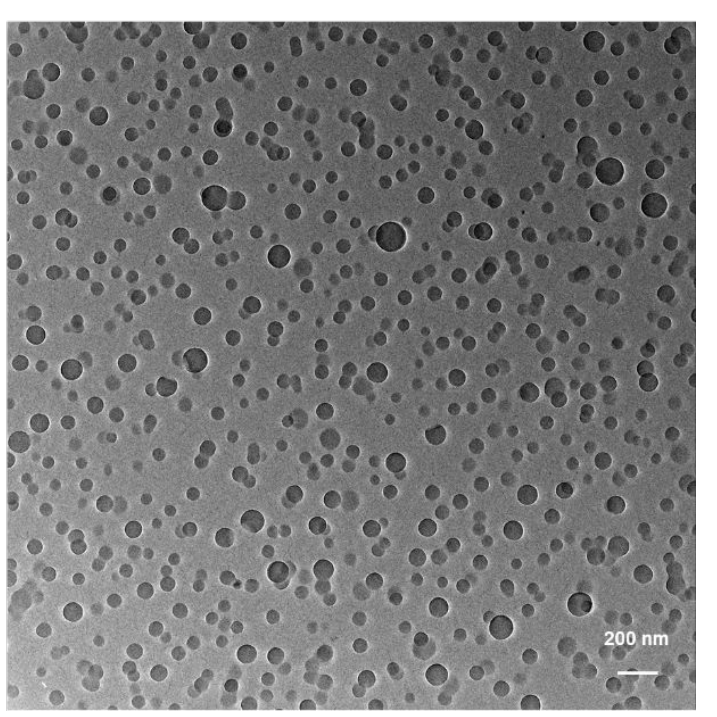

b)

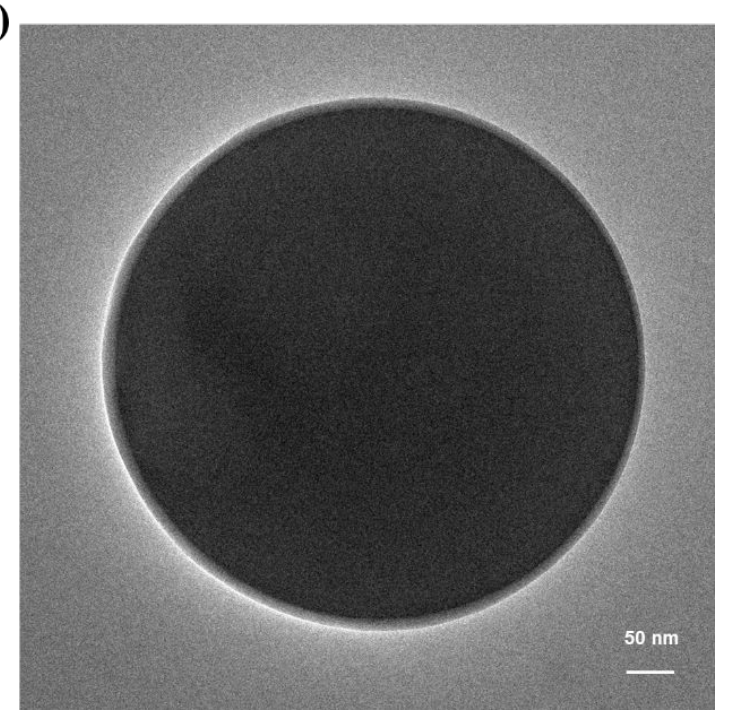

Figure S3: Representative TEM images of COP-5T after polymerization for $6 \mathrm{~h}$ (a) and $12 \mathrm{~h}$ (b). 


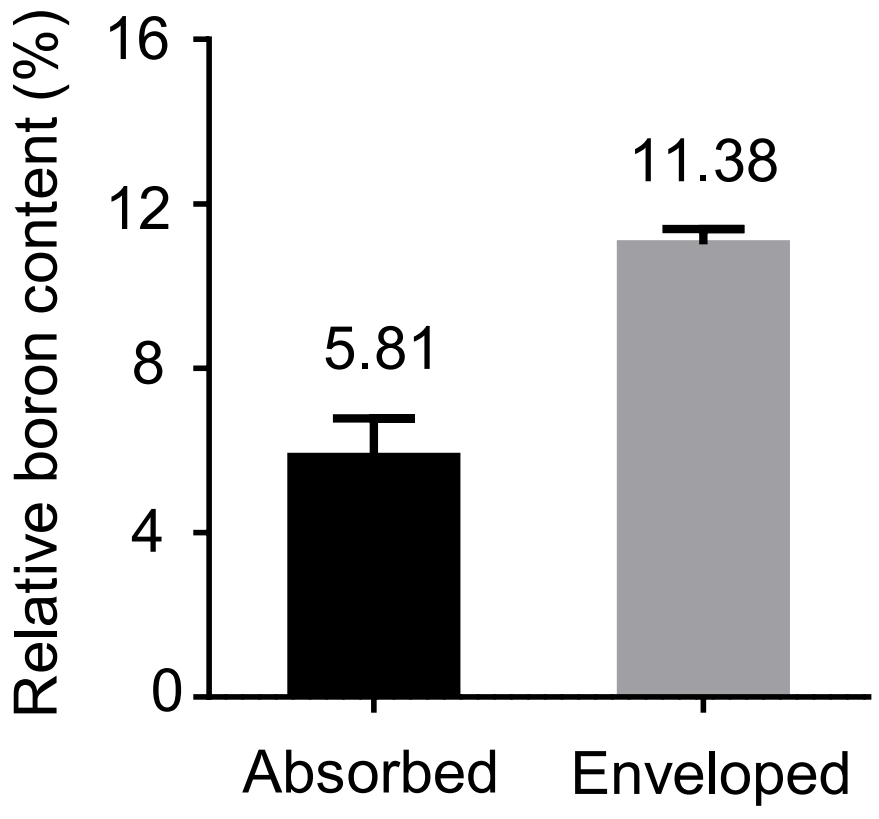

Figure S4. Boron concentration of drugs prepared by two methods: the adsorption method after synthesis of COP-5T and the enveloped method before the polymerization. Relative boron content refers to weight ratio for carborane: COP-5T. 


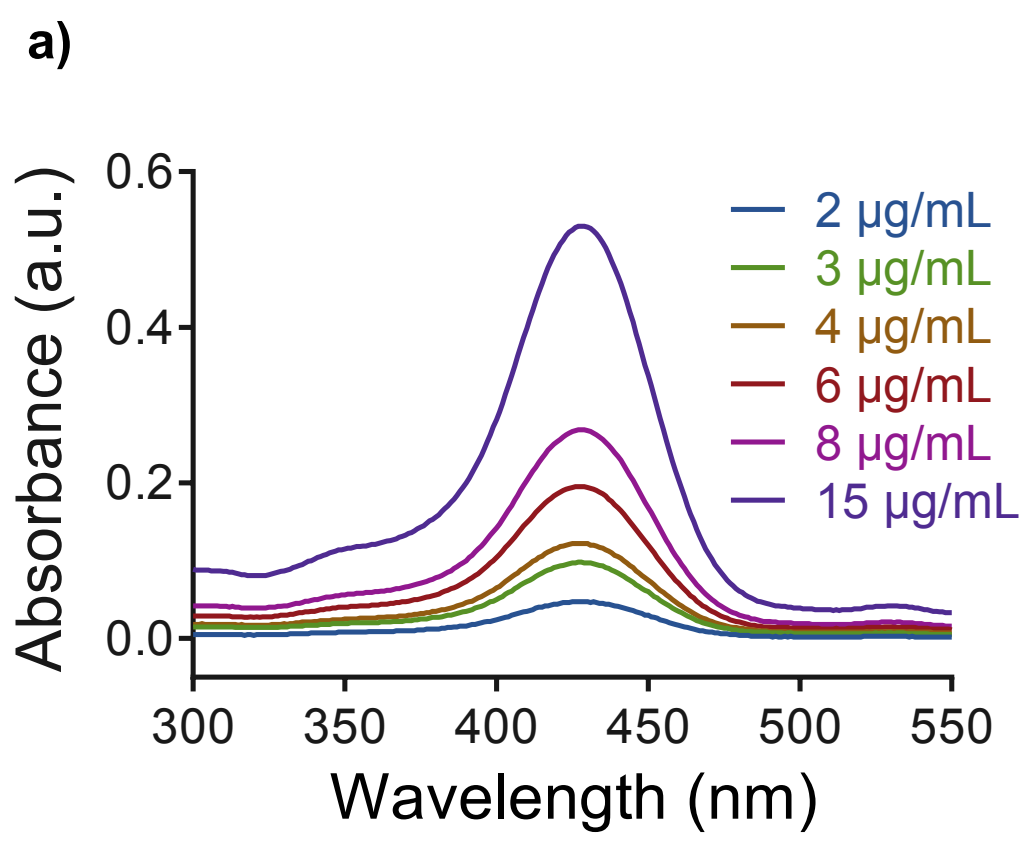

b)

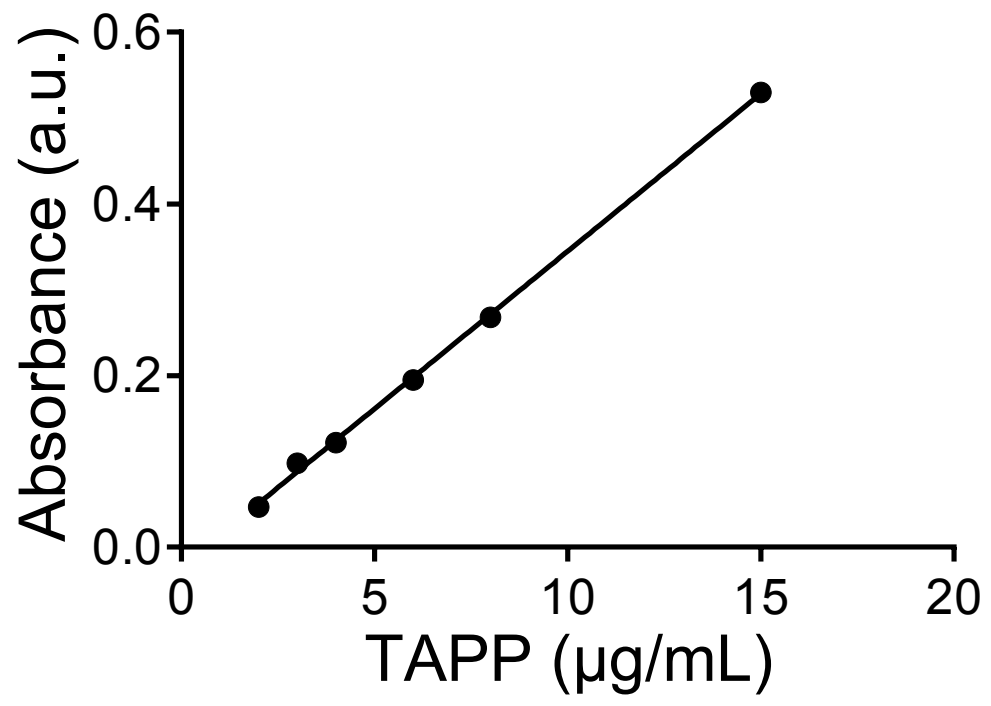

Figure S5. UV-Vis absorption spectra of COP-5T (a) and its UV standard curve (b) at variant concentrations. (Concentrations were determined by TAPP from $0.01 \mathrm{mg}$ $\mathrm{mL}^{-1}$ to $\left.0.1 \mathrm{mg} \mathrm{mL}^{-1}\right)$. 


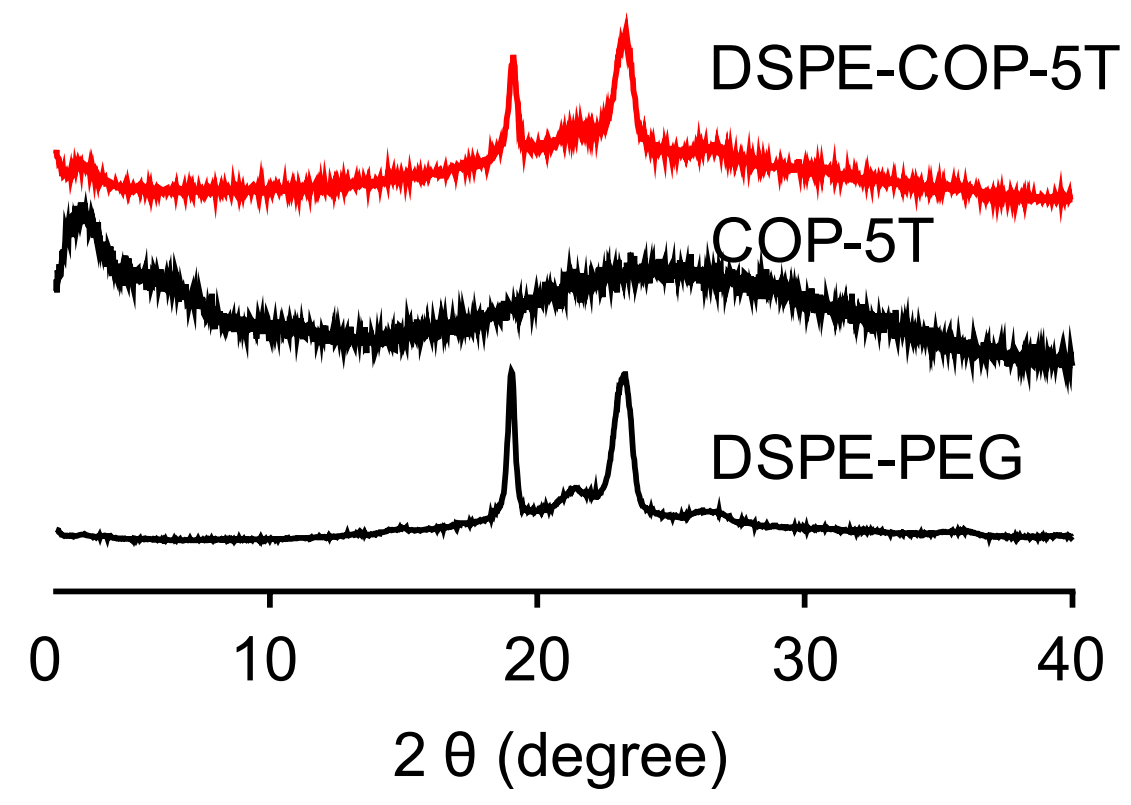

Figure S6. Powder X-Ray Diffraction of DSPE-COP-5T, COP-5T and DSPE-PEG. 


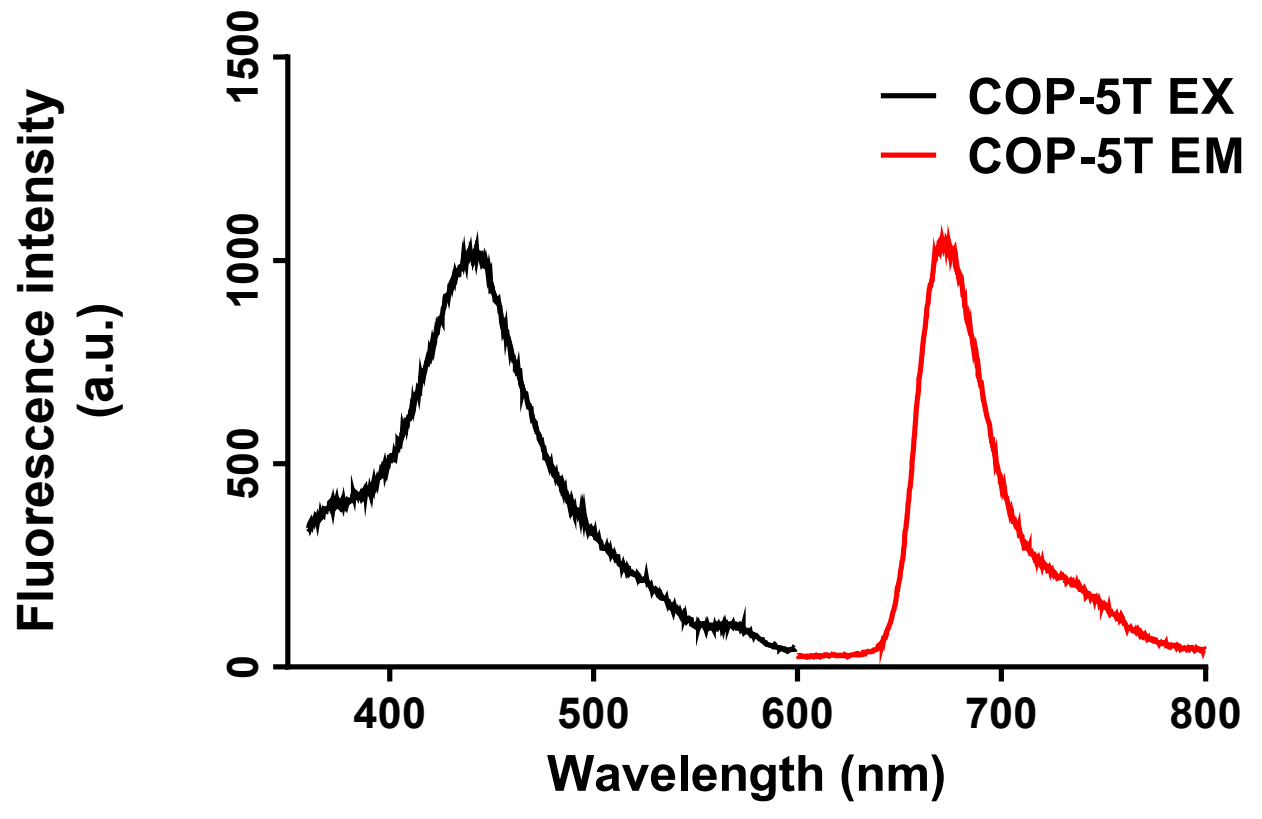

Figure S7. Fluorescence spectrum of COP-5T. 


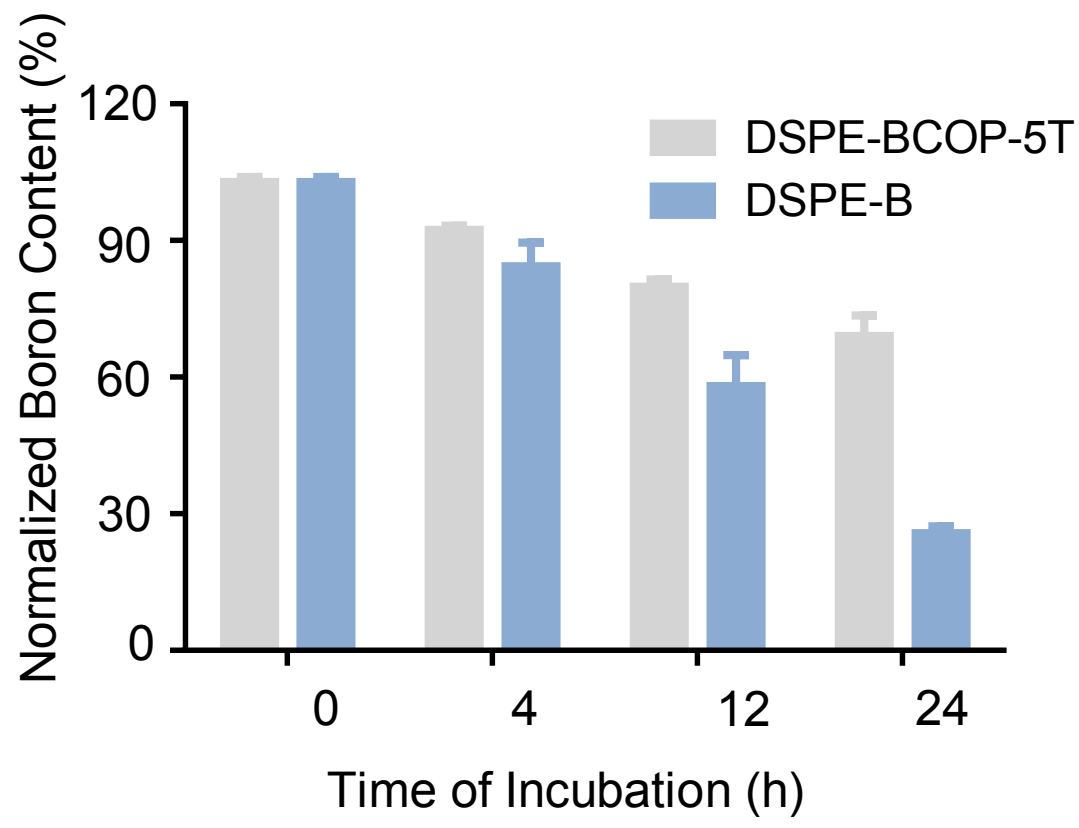

Figure S8. The relative boron content of DSPE-BCOP-5T (gray) and DSPE-B (blue) after incubation in fetal bovine serum (FBS) for different times $(n=6)$. 

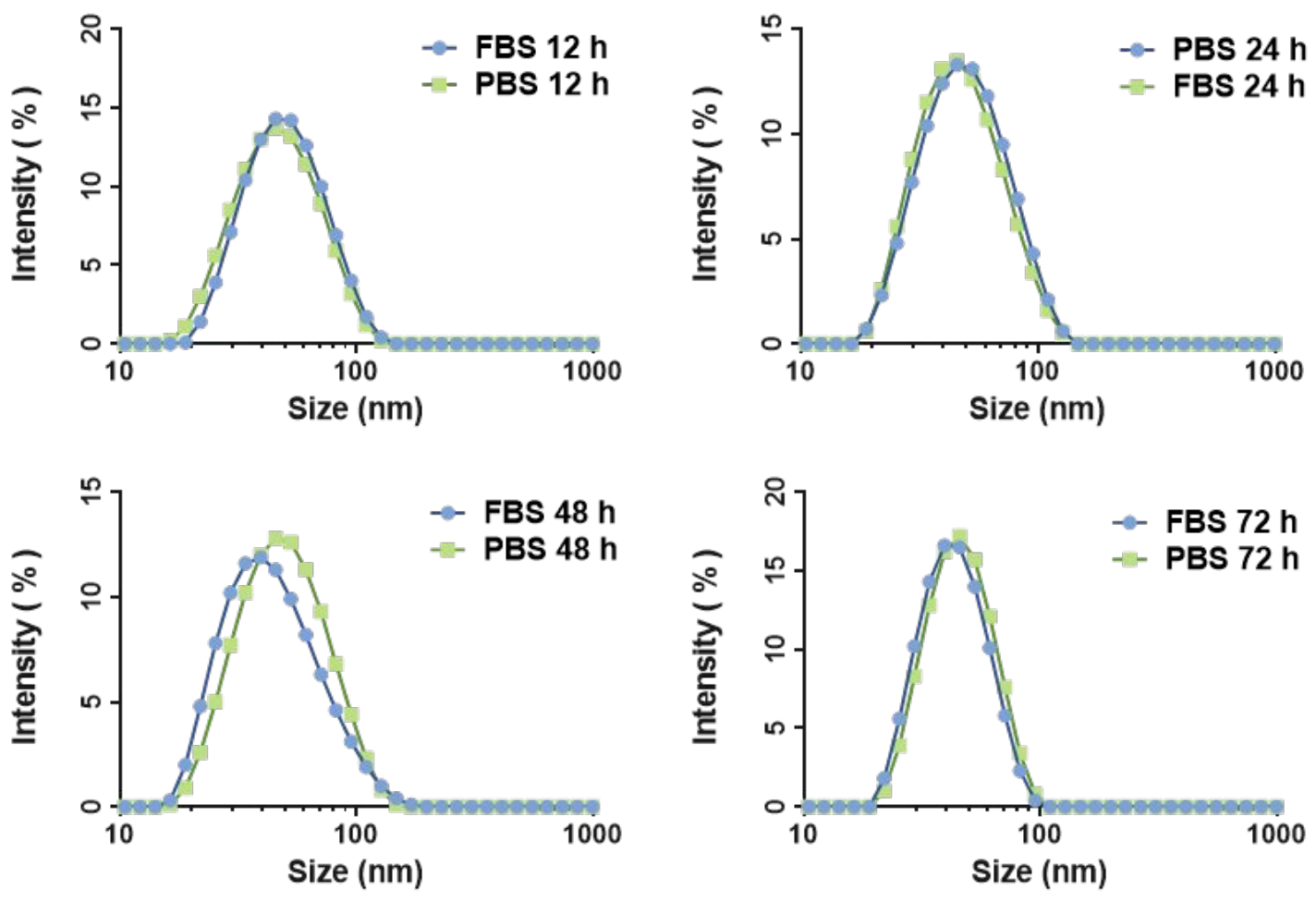

Figure S9. Particle size in PBS and FBS solutions remained stable. 

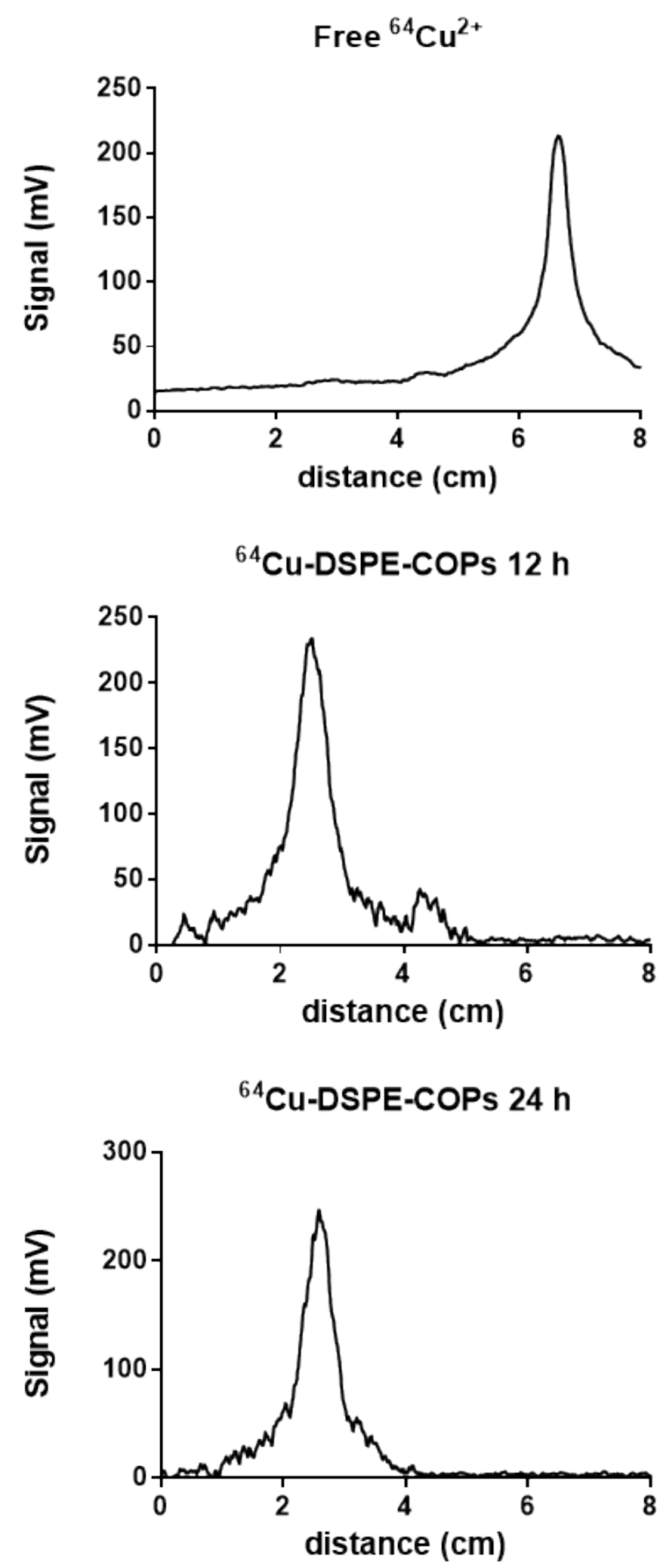

Figure S10. Radio-TLC of free ${ }^{64} \mathrm{Cu}-\mathrm{Cu}^{2+}$ and ${ }^{64} \mathrm{Cu}-\mathrm{DSPE} @ \mathrm{COP}-5 \mathrm{~T}$ after the incubation in serum for 12 and 24 hours. 


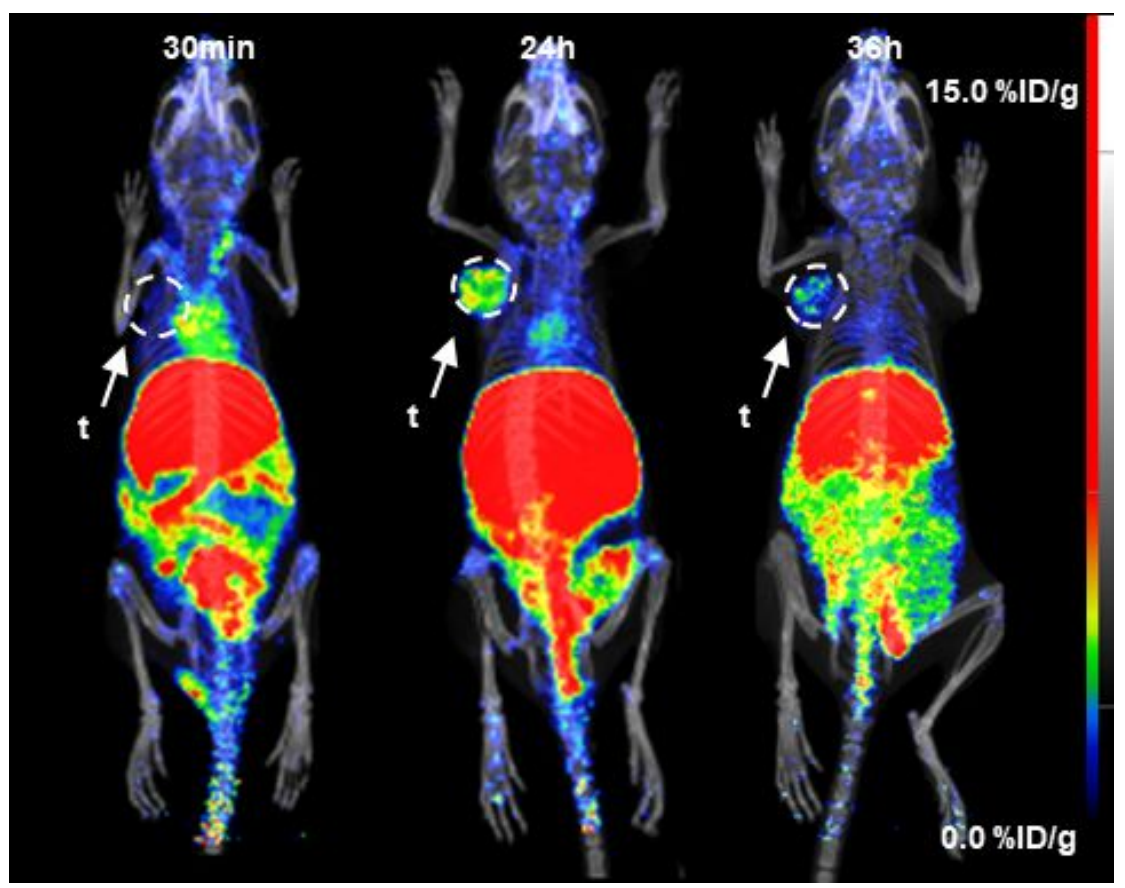

Figure S11. Representative whole-body maximum intensity projection PET images of ${ }^{64} \mathrm{Cu}-\mathrm{DSPE}-\mathrm{BCOP}-5 \mathrm{~T}$ at certain time points after tail-veil injection $(\mathrm{n}=3)$. The tumors (t) are indicated by white arrows. 

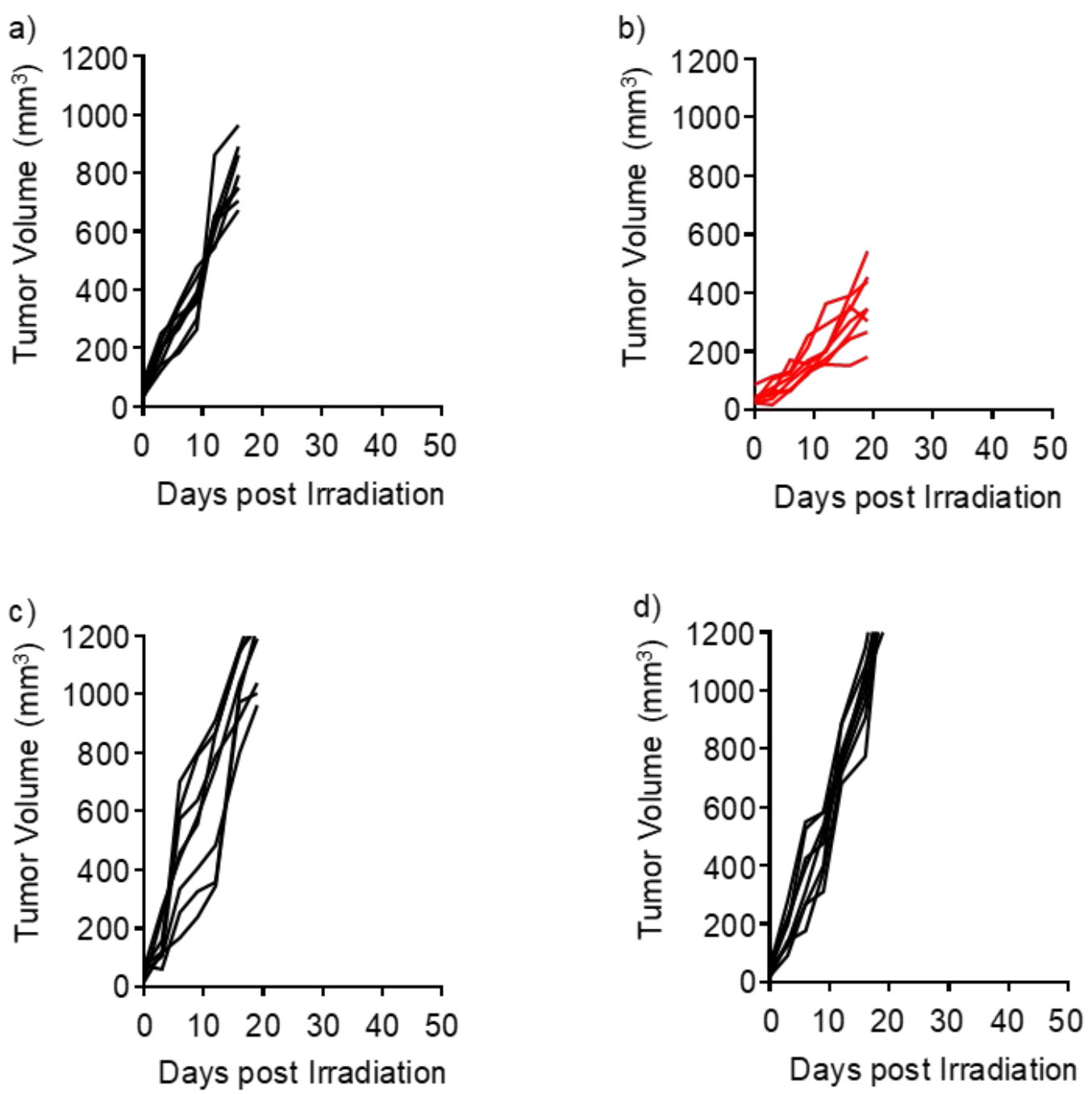

Figure S12. Representative photographs of each group of 4T1 tumor bearing mice days post treatment ( $\mathrm{n}=8)$. (a) Neutron only group. (b) DSPE-BCOP-5T + neutron group. (c) Control group. (d) DSPE-BCOP-5T only group. 
a)

b)
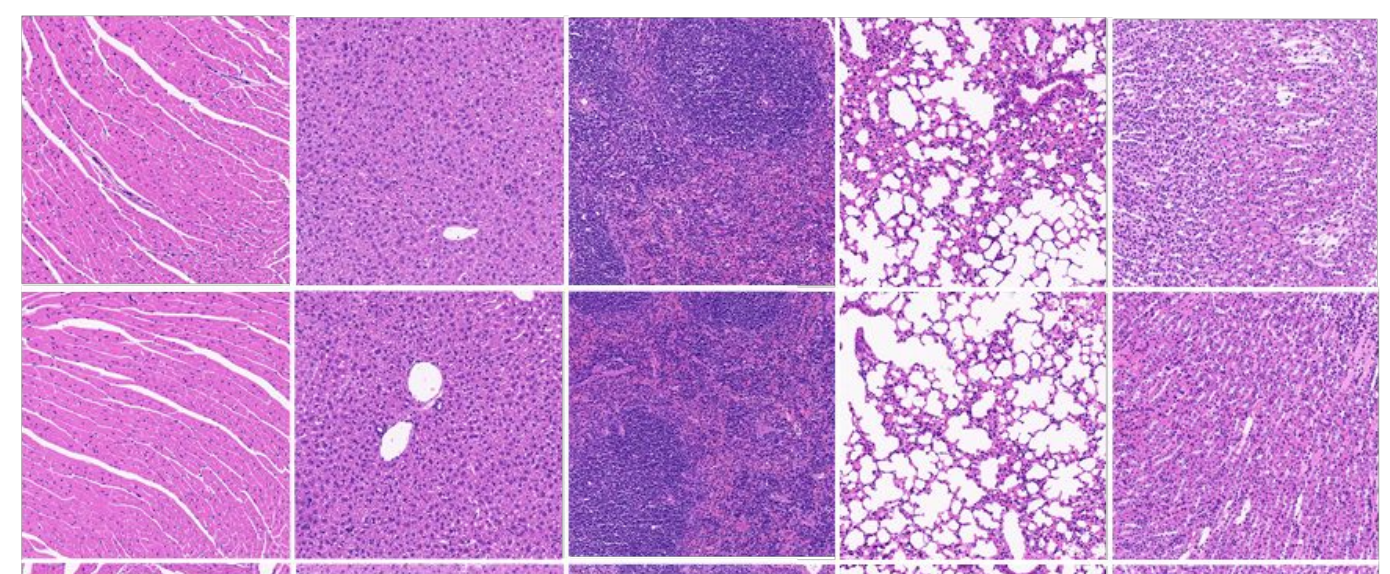

c)
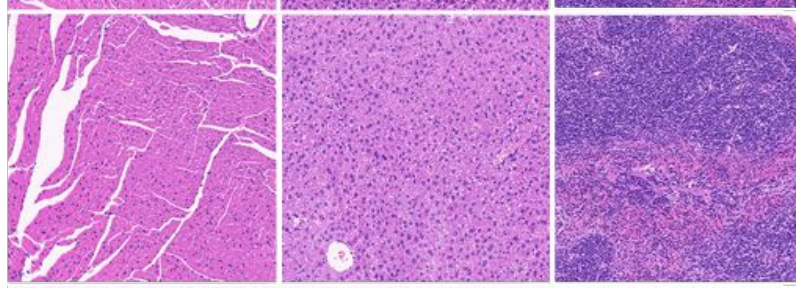

d)
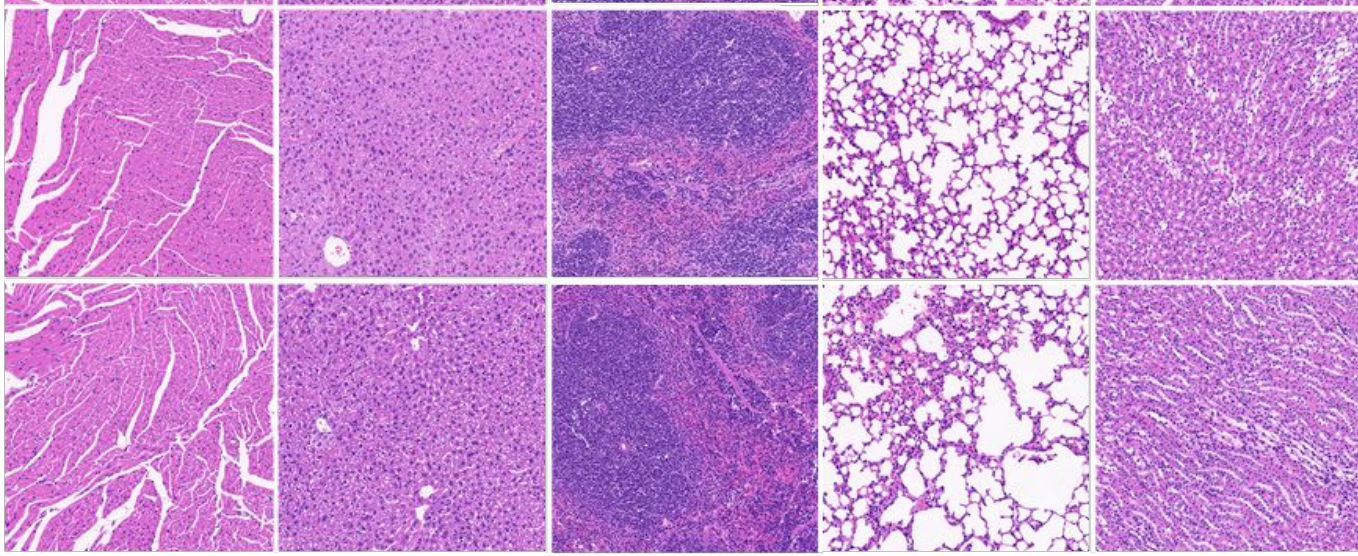

Figure S13. Hematoxylin-eosin tained (H\&E) of heart, liver, spleen, lung and kidney slices collected from different groups of mice 20 days after various treatments. (a) Neutron only group. (b) DSPE-BCOP-5T + neutron group. (c) Control group. (d) DSPE-BCOP-5T only group. 
Table S1. Biodistribution data of ${ }^{64} \mathrm{Cu}-\mathrm{DSPE}-\mathrm{COP}-5 \mathrm{~T}$ in $4 \mathrm{~T} 1$ tumor bearing mice $24 \mathrm{~h}$ post once injection $(n=6)$.

\begin{tabular}{ccc}
\hline Tissues & Means \%ID/g & RSD \\
\hline Blood & 5.32 & 1.54 \\
Liver & 27.29 & 3.78 \\
Spleen & 7.96 & 0.42 \\
Kidney & 8.86 & 1.06 \\
Intestine & 6.75 & 3.29 \\
Fat & 0.73 & 0.20 \\
Bone & 3.13 & 0.85 \\
Pancreas & 5.38 & 1.30 \\
Muscle & 1.15 & 0.11 \\
Brain & 1.03 & 0.09 \\
Tumor & 7.95 & 0.58 \\
\hline
\end{tabular}


Table S2. Biodistribution of DSPE-BCOP-5T in $4 \mathrm{~T} 1$ bearing mice $24 \mathrm{~h}$ post single bolus injection $(n=6)$ and three separated injections $(n=6)$.

\begin{tabular}{ccccc}
\hline & \multicolumn{2}{c}{ Single bolus injection } & \multicolumn{2}{c}{ Three separated injections } \\
Tissues & Boron content $(\mu \mathrm{g} / \mathrm{g})$ & RSD & Boron content $(\mu \mathrm{g} / \mathrm{g})$ & RSD \\
\hline Blood & 42.56 & 3.14 & 13.82 & 0.62 \\
Liver & 231.06 & 5.28 & 115.35 & 4.96 \\
Spleen & 81.27 & 2.07 & 24.78 & 0.67 \\
Kidney & 70.17 & 2.14 & 16.27 & 1.78 \\
Intestine & 166.78 & 3.13 & 23.93 & 2.30 \\
Fat & 6.91 & 0.04 & 2.18 & 0.68 \\
Bone & 12.33 & 2.16 & 5.18 & 1.49 \\
Pancreas & 71.75 & 0.06 & 27.36 & 3.83 \\
Muscle & 37.80 & 2.08 & 3.39 & 0.22 \\
Brain & 8.40 & 0.01 & 4.15 & 0.72 \\
Tumor & 55.24 & 2.13 & 84.93 & 2.68 \\
\hline
\end{tabular}




\section{REFERENCES}

1. Duan, D.; Liu, H.; Xu, Y.; Han, Y.; Xu, M.; Zhang, Z.; Liu, Z., Activating TiO2 nanoparticles: gallium-68 serves as a high-yield photon emitter for Cerenkov-induced photodynamic therapy. ACS Appl. Mater. Interfaces 2018, 10 (6), 5278-5286.

2. Chen, J.; Li, C.; Hong, H.; Liu, H.; Wang, C.; Xu, M.; Han, Y.; Liu, Z., Side Chain Optimization Remarkably Enhances the in Vivo Stability of 18F-Labeled Glutamine for Tumor Imaging. Mol. Pharmaceutics 2019, 16, 5035-5041. 